\title{
MLSTar: automatic multilocus sequence typing of bacterial genomes in $\mathbf{R}$
}

\author{
Ignacio Ferrés $^{1}$, Gregorio Iraola ${ }^{\text {Corresp. }{ }^{1,2}}$ \\ 1 Bioinformatics Unit, Institut Pasteur de Montevideo, Montevideo, Uruguay \\ 2 Center for Integrative Biology, Universidad Mayor, Santiago de Chile, Chile \\ Corresponding Author: Gregorio Iraola \\ Email address: giraola@pasteur.edu.uy
}

Multilocus sequence typing (MLST) is a standard tool in population genetics and bacterial epidemiology that assesses the genetic variation present in a reduced number of housekeeping genes (typically seven) along the genome. This methodology assigns arbitrary integer identifiers to genetic variations at these loci allowing to efficiently compare bacterial isolates using allele-based methods. Now, the increasing availability of whole-genome sequences for hundreds to thousands of strains from the same bacterial species has allowed to apply and extend MLST schemes by automatic extraction of allele information from the genomes. The PubMLST database is the most comprehensive resource of described schemes available for a wide variety of species. Here we present MLSTar as the first R package that allows to i) connect with the PubMLST database to select a target scheme, ii) screen a desired set of genomes to assign alleles and sequence types and iii) interact with other widely used $\mathrm{R}$ packages to analyze and produce graphical representations of the data. We applied MLSTar to analyze more than 2500 bacterial genomes from different species, showing great accuracy and comparable performance with previously published command-line tools. MLSTar can be freely downloaded from http://github.com/iferres/MLSTar. 


\title{
MLSTar: automatic multilocus sequence typing of bacterial genomes in $\mathbf{R}$
}

\section{Ignacio Ferrés ${ }^{1}$ and Gregorio Iraola ${ }^{1,2}$}

\author{
${ }^{1}$ Bioinformatics Unit, Institut Pasteur de Montevideo, Montevideo, Uruguay \\ ${ }^{2}$ Center for Integrative Biology, Universidad Mayor, Santiago de Chile, Chile \\ Corresponding author: \\ Gregorio Iraola ${ }^{1}$ \\ Email address: giraola@pasteur.edu.uy
}

\begin{abstract}
Multilocus sequence typing (MLST) is a standard tool in population genetics and bacterial epidemiology that assesses the genetic variation present in a reduced number of housekeeping genes (typically seven) along the genome. This methodology assigns arbitrary integer identifiers to genetic variations at these loci allowing to efficiently compare bacterial isolates using allele-based methods. Now, the increasing availability of whole-genome sequences for hundreds to thousands of strains from the same bacterial species has allowed to apply and extend MLST schemes by automatic extraction of allele information from the genomes. The PubMLST database is the most comprehensive resource of described schemes available for a wide variety of species. Here we present MLSTar as the first R package that allows to i) connect with the PubMLST database to select a target scheme, ii) screen a desired set of genomes to assign alleles and sequence types and iii) interact with other widely used $R$ packages to analyze and produce graphical representations of the data. We applied MLSTar to analyze more than 2500 bacterial genomes from different species, showing great accuracy and comparable performance with previously published command-line tools. MLSTar can be freely downloaded from http://github. com/iferres/MLSTar.
\end{abstract}

\section{INTRODUCTION}

Multilocus sequence typing (MLST) was introduced in 1998 as a portable tool for studying epidemiological dynamics and population structure of bacterial pathogens based on PCR amplification and capillary sequencing of housekeeping gene fragments (Maiden et al. 1998). In most MLST schemes, seven loci are indexed with arbitrary and unique allele numbers that are combined into an allelic profile or sequence type (ST) to efficiently summarize genetic variability along the genome. Rapidly, MLST demonstrated enhanced reproducibility and convenience in comparison with previous methods such as multilocus enzyme electrophoresis (MLEE) or pulsed-field gel electrophoresis (PFGE), allowing to perform global epidemiology and surveillance studies (Urwin and Maiden, 2003). For example, MLST has been applied to elucidate the global epidemiology of Burkholderia multivorans in cystic fibrosis patients (Baldwin et al. 2008) or to understand the dissemination of antibiotic-resistant enterobacteria (Castanheira et al. 2011). However, as MLST started to be massively applied two main drawbacks were uncovered: i) the impossibility of establishing a single universal MLST scheme applicable to all bacteria; and ii) the lack of high resolution of seven-locus MLST schemes required for some purposes.

These problems pushed the development of improved alternatives to the original methodology. The extended MLST (eMLST) approach which is based on the analysis of longer gene fragments (Chen et al. 2011) or increased number of loci (Dingle et al. 2008: Crisafulli et al. 2013) proved to improve resolution, and the scheme based on 53 ribosomal protein genes (rMLST) was proposed as an universal approach since these loci are conserved in all bacteria (Jolley et al. 2012). Beyond these improvements, the advent of high-throughput sequencing and the increasing availability of hundreds to thousands whole-genome sequences (WGS) for many bacterial pathogens caused a paradigmatic change in clinical microbiology, making possible to use nearly complete genomic sequences to enhance typing resolution. This revolution allowed the transition from standard MLST schemes testing a handful of genes to core genome (cgMLST) 
approaches that scaled to hundreds of loci common to a set of bacterial genomes (Maiden et al. 2013).

The generation of this massive amount of genetic information required the accompanying development of database resources to effectively organize and store typing schemes and allele definitions. Rapidly, the PubMLST database (http://pubmlst.org) turned into the most comprehensive and standard resource storing today schemes and allelic definitions for more than 100 microorganisms. Subsequently, the shift to WGS motivated the development of the Bacterial Isolate Genome Sequence Database (BIGSdb) (Jolley and Maiden, 2010), which now encompasses all the software functionalities used for the PubMLST. Also, many tools for automatic MLST analysis from wholegenome sequences have been developed using web servers like MLST-OGE (Larsen et al. 2012) or EnteroBase (http: //enterobase.warwick.ac.uk), pay-walled tools like BioNumerics or SeqSphere+, and open source tools like mlst (http://github.org/tseemann/mlst) or MLST check (Page et al. 2016). Here, we present MLSTar as the first tool for automatic multilocus sequence typing of bacterial genomes written in $\mathrm{R}$ (R Development Core Team 2008), allowing to expand the application of MLST tools within this very popular and useful environment for data analysis and visualization.

\section{METHODS}

\section{Implementation}

MLSTar is written in R and contains all data processing steps and command line parameters to call external dependencies wrapped in the package. MLSTar depends on BLAST+ (Camacho et al., 2009) that is used as sequence search engine, and must be installed locally. MLSTar is designed to work on Unix-based operating systems and is distributed as an open source software (MIT license) stored in GitHub (http://github.com/iferres/MLSTar). MLSTar contains four main functions that i) takes genome assemblies or predicted genes in FASTA format from any number of strains, ii) performs sequence typing using a previously selected scheme from PubMLST and iii) applies standard phylogenetic approaches to analyze the data. An overview of the overall workflow has been outlined in Figure 1 .

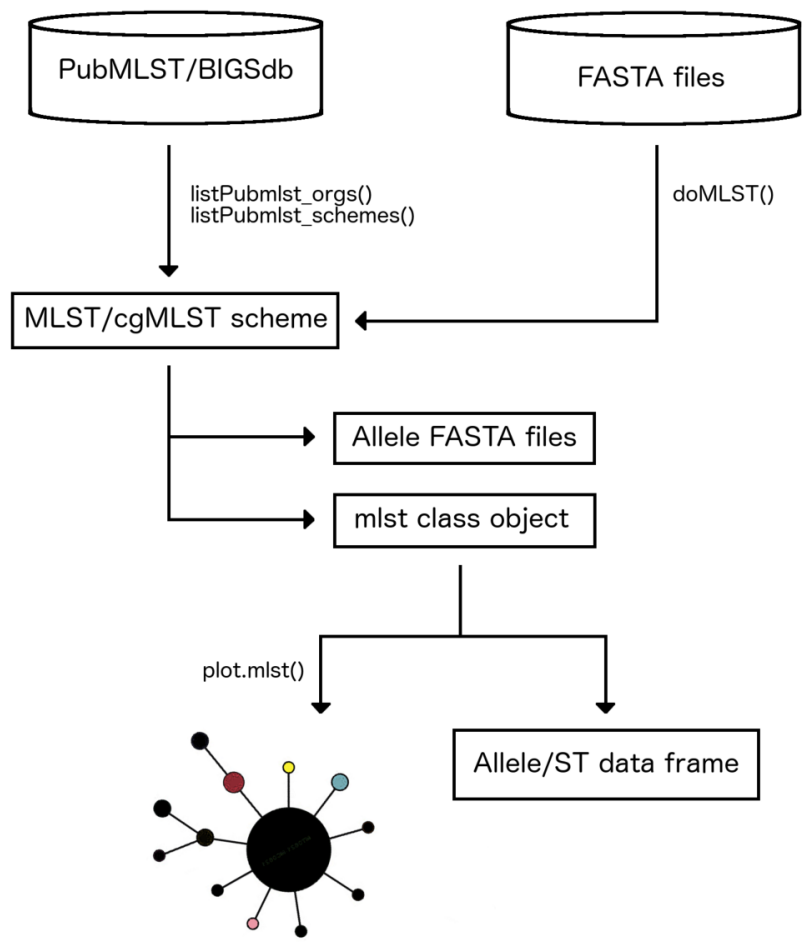

Figure 1. Main steps in MLSTar workflow. 


\section{Interaction with PubMLST}

First step in MLSTar workflow involves to interact with the PubMLST database to select a target scheme. This interaction requires Internet connection because is performed using the RESTful web application programming interface provided by PubMLST. The listPubmlst_orgs () function allows to list the names of all microorganisms that have any scheme stored in PubMLST. Then, as some microorganisms have more than one scheme (i.e. one classical seven-loci and one core genome scheme), the listPubmlst_schemes () function lists the available schemes for any selected species. Additionally, MLSTar is not restricted only to the MLST definitions present in PubMLST since schemes stored in other databases can be manually downloaded and analyzed with MLSTar.

\section{Calling and storing alleles and sequence types}

MLSTar make allele and ST calls from FASTA files containing closed genomes or contigs using BLAST+ blastn comparisons implemented by the doMLST ( ) function. Parallelization is available as internally implemented in R by the parallel package. Also, the doMLST () function can be run at the same time for different schemes using internal R functions like lapply (). Results are stored in a S3 class object named mlst that contains two data. frame objects: one containing allele and ST assignments for the analyzed genomes (unknown alleles or STs are labeled as "u"), and the other storing known allele profiles for the selected scheme. If required, nucleotide sequences for known or novel alleles can be written as multi FASTA files for downstream analyses.

\section{Post analysis}

Allele profiles are frequently used to reconstruct phylogenetic relationships among strains. Function plot.mlst () directly takes the mlst class object to compute distances assuming no relationships between allele numbers, so each locus difference is treated equally. Then, identical isolates have a distance of 0 , those with no alleles in common have a distance of 1 and, for example, in a seven-loci scheme two strains with 5 differences would have a distance of $0.71(5 / 7)$. The resulting distance matrix is used to build a minimum spanning tree using igraph (Csardi and Nepusz, 2006) that returns an object of class igraph or a neighbor-joining tree as implemented in APE package (Paradis et al. 2004) that returns an object of class phylo. The package also contains a specific method defined as plot.mlst that recognizes the mlst class object and plots the results using the generic plot () function. Additionally, a better resolution analysis based on the variability of the underlying sequences using more sophisticated Maximum-Likelihood or Bayesian phylogenies, can be achieved externally by aligning the allele sequences that are automatically retrieved by MLSTar.

\section{RESULTS AND DISCUSSION}

\section{Comparison with capillary sequencing data}

MLST analysis based on capillary sequencing has been considered as the gold standard. Hence, we used a previously reported dataset (Page et al., 2017) consisting in 72 Salmonella samples originally tested by capillary sequencing and deposited in the EnteroBase (Alikhan et al., 2018), that were posteriorly whole-genome sequenced. This dataset covers a wide host range and isolation dates of Salmonella strains comprising 32 different STs (Supplemental Table S1). In average, MLSTar assignments at ST level matched in $92 \%$ of cases when compared with capillary sequencing. Additionally, ST calls for five samples that were distinct between capillary sequencing and genome-derived inferences using several software tools (Page et al. 2017), were also discordant in the same way when using MLSTar. This is expected since capillary sequencing is not error free (Liu et al. 2012), in spite of being considered as the gold standard. By the contrary, the result for sample $139 \mathrm{~K}$ matched between capillary sequencing and MLST ar but most other software tools, except stringMLST (Gupta et al. 2016), failed to assign confident STs. MLSTar results on the same dataset but in comparison with other softwares designed to screen whole-genome assemblies such as mlst (http://github.org/t seemann/mlst) and MLSTcheck (Page et al. 2016) matched in $89 \%$ and $92 \%$ of cases, respectively. These results demonstrate that MLSTar and other software have comparable performance when testing against standard MLST results based on capillary sequencing. 


\section{Comparison against BIGSdb}

We retrieved 2726 genomes from the BIGS db belonging to 10 species most of which are very well-known pathogens (Supplemental Table S2). For these datasets, reference allele and ST assignments based on the corresponding standard MLST schemes were extracted from the BIGS db and compared with results obtained running MLSTar. The concordance at allele and ST levels is shown in Table 1, measured as the percentage of identical assignments between BIGSdb and MLSTar. In average, assignments were $97.9 \%(\mathrm{SD}=1.95)$ and $95.6 \%(\mathrm{SD}=2.5)$ coincident for alleles and STs, respectively. These results evidence a very good performance of MLSTar in comparison with the reference assignments from the BIGSdb. Additionally, we tested MLSTar using the ribosomal MLST scheme (Jolley et al. 2012) over the same 354 genomes belonging to Staphylococcus aureus and Streptococcus agalactiae. This scheme was conceived as an universal approach for discrimination of bacterial species. Accordingly, the automatic phylogenetic analysis implemented in MLSTar was able to discriminate both species using ribosomal alleles (Fig. 2).

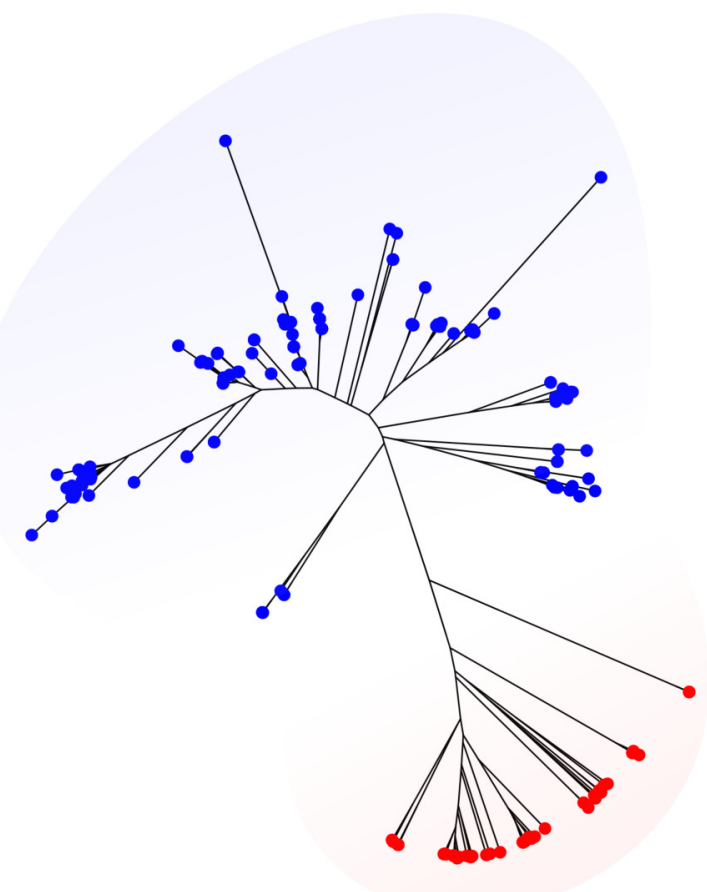

Figure 2. Phylogeny based on ribosomal alleles. Staphylococcus aureus (red) and Streptococcus agalactiae (blue) genomes from the BIGSdb $(n=356)$ were characterized using the universal rMLST scheme (based on 53 ribosomal genes). The phylogenetic tree was automatically generated with the plot.mlst ( ) function using the Neighbor-Joining algorithm from a distance matrix obtained from allele patterns.

\section{Comparison with MLST schemes of close species}

The PubMLST database stores schemes for 10 different species within the genus Campylobacter, hence we used this case as negative control to test the specificity of MLSTar. We chose the 172-C.jejuni/coli dataset from BIGSdb and 150 randomly selected $C$. fetus genomes from a previously published study (Iraola et al. 2017) to run MLS Tar against the schemes defined for the remaining Campylobacter species, in order to detect potential false positive calls when analyzing closely related taxa. False positives at both allele and ST levels were not detected neither for $C$. jejuni/coli nor for $C$. fetus against the rest (Supplemental Table S3), indicating that MLSTar is highly specific when working with genetically related bacteria. 
Table 1. Accuracy of MLSTar against reference alleles and STs obtained from BIGSdb, measured as the percentage of correct calls in seven-locus MLST schemes from 11 different pathogens comprising a total of 3,021 genomes.

\begin{tabular}{|c|c|c|c|c|c|c|c|c|c|}
\hline Species & Genomes & Scheme & & & & & & & \\
\hline Bordetella snn & 66 & $a d k$ & fumC & $\operatorname{gly} A$ & tyrB & icd & pepA & pgm & ST \\
\hline Boraetella spp. & 06 & 96.7 & 96.7 & 96.7 & 96.7 & 96.7 & 95 & 96.7 & 95 \\
\hline Stanbylococouc aurous & 77 & $g d h$ & gyd & $p s t S$ & $g k i$ & aroE & $x p t$ & yqiL & ST \\
\hline stapnylococcus aureus & 12 & 94.4 & 94.4 & 94.5 & 95.3 & 94.4 & 95.2 & 99.4 & 93.1 \\
\hline Holichlactor nulori & 70 & atpA & $e f p$ & mutY & $p p a$ & $\operatorname{trpC}$ & ureI & $y p h C$ & ST \\
\hline Hellcobacter pyiort & 19 & 97.5 & 96.2 & 98.7 & 97.5 & 98.7 & 97.5 & 97.5 & 93.7 \\
\hline Darillue ormor & 115 & $g l p$ & gmk & $i l v$ & pta & pur & pyc & tpi & ST \\
\hline Bactinus cereus & 115 & 98.3 & 100 & 100 & 100 & 100 & 96.5 & 98.2 & 93.9 \\
\hline Campvlohacter ieiuni/coli & 176 & aspA & $g \ln A$ & gltA & glyA & pgm & $t k t$ & uncA & ST \\
\hline Сатруловастег јејиплсол & & 100 & 99 & 100 & 100 & 100 & 100 & 100 & 99 \\
\hline Burkholderia ns & 225 & ace & glt $B$ & $g m h D$ & lepA & lipA & narK & $n d h$ & ST \\
\hline & & 98.7 & 96 & 93 & 96 & 96.9 & 95.6 & 96 & 93 \\
\hline Streptococcus asalactiae & 258 & adhP & pheS & atr & $g \ln A$ & $s d h A$ & glcK & $t k t$ & ST \\
\hline & 250 & 99.2 & 99.6 & 99.2 & 99.2 & 99.2 & 99.6 & 99.6 & 98.1 \\
\hline Klebsiella pneumoniae & 284 & $\overline{\text { gapA }}$ & inf $B$ & $m d h$ & $p g i$ & phoE & rpoB & tonB & ST \\
\hline & 284 & 100 & 100 & 100 & 100 & 100 & 100 & 100 & 100 \\
\hline Pseudomonas aeruginosa & 604 & acs & aro & gua & mut & пио & $p p s$ & trp & ST \\
\hline г senaomonas aeruginosa & 004 & 96.4 & 98.8 & 98.1 & 98.3 & 98.1 & 98.3 & 98.8 & 95.9 \\
\hline Acinetobacter baumannii & 847 & cpn60 & fusA & gltA & pyrG & recA & rplB & rpoB & ST \\
\hline & $0+1$ & 98.6 & 97.4 & 99.3 & 99.2 & 97.3 & 99.1 & 98.7 & 94.9 \\
\hline
\end{tabular}

A

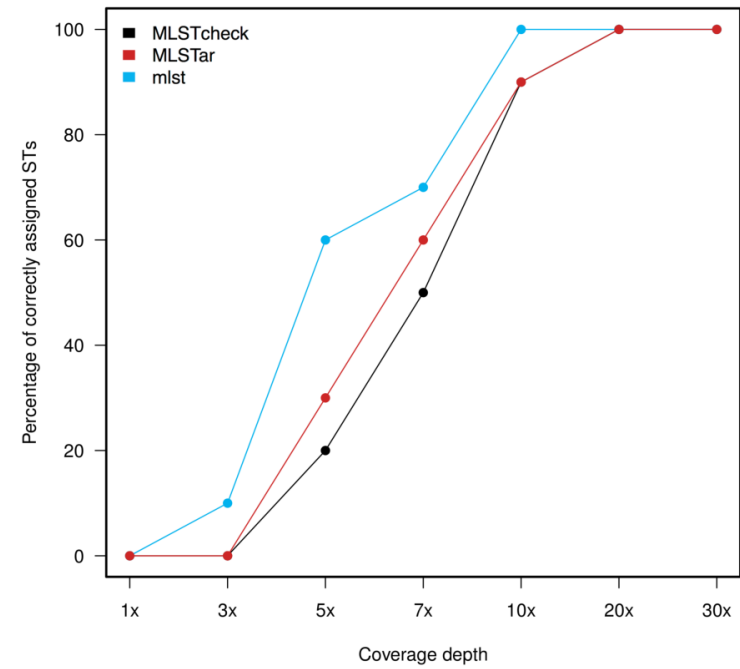

B

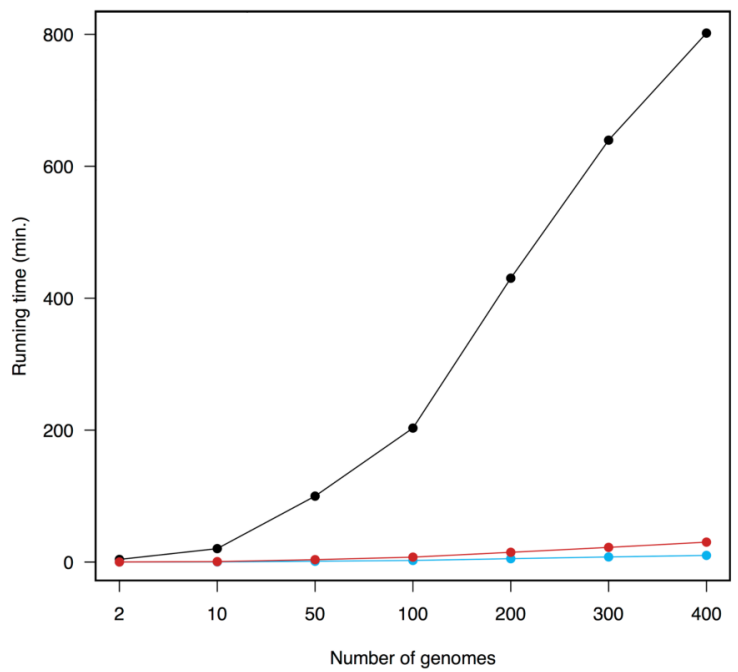

Figure 3. Comparison of MLSTar performance. A) Comparison of MLSTar, MLSTcheck and mlst softwares using a dataset of 10 Salmonella genomes de novo assembled at variable coverage depths. B) Comparison of MLSTar, MLSTcheck and ml st running times on a single CPU using increasing number of genomes.

\section{Comparison of variable coverage depths and number of genomes}

Variable depths of sequencing coverage have been shown to affect the accuracy of different softwares to achieve confident ST calls. In general, most softwares require over than 10x to ensure optimal performance (Page et al. 2017). Here, we tested MLSTar by sampling reads at gradual depths from 10 genomes (representing different STs) from the Salmonella dataset and measured the percentage of correctly assigned 
STs. Figure $3 \mathrm{~A}$ shows that MLSTar produce good-enough results when sequencing depth is greater than 10x, and its performance is comparable to similar tools such as MLSTcheck and mlst. Considering that nowadays bacterial genome sequencing experiments typically ensure at least 30x of coverage depth, our results evidence that MLSTar is appropriate for analyzing whole-genome sequences with average or even slightly lower coverage depths. Additionally, we used a random set of genomes $(n=400)$ from the BIGSdb dataset to compare the running time between MLSTar, MLSTcheck and mlst softwares in a single AMD Opteron $2.1 \mathrm{GHz}$ processor, by gradually increasing the number of analyzed genomes from 2 to 400 (Fig. 3B). These results showed that MLSTar is 26-fold faster than MLSTcheck but is 3-fold slower than mlst (Supplemental Table S4).

\section{CONCLUSIONS}

The advent of WGS has now allowed to type bacterial strains directly from their whole genomes avoiding to repeat tedious PCR amplifications and fragment capillary sequencing for multiple loci. Today MLST is a valid tool which is frequently used as first-glimpse approach to explore genetic diversity and structure within huge bacterial population sequencing projects. This incessant availability of genomic information has motivated a constant effort to develop efficient analytical tools from multilocus typing data (Page et al. 2017). Here, we developed a new software package called MLSTar that expands the possibilities of performing allele-based genetic characterization within the R environment. We demonstrate that MLSTar has comparable performance with previously validated software tools and can be applied to analyze hundreds of genomes in a reasonable time.

\section{ACKNOWLEDGMENTS}

We thank Daniela Costa and Cecilia Nieves for testing MLSTar.

\section{REFERENCES}

Alikhan, N., Zhou, Z., Sergeant, M., and Achtman, M. (2018). A genomic overview of the population structure of salmonella. PLoS genetics, 14(4):e1007261.

Baldwin, A., Mahenthiralingam, E., Drevinek, P., Pope, C., Waine, D. J., Henry, D. A., Speert, D. P., Carter, P., Vandamme, P., LiPuma, J. J., et al. (2008). Elucidating global epidemiology of burkholderia multivorans in cases of cystic fibrosis by multilocus sequence typing. Journal of clinical microbiology, 46(1):290-295.

Camacho, C., Coulouris, G., Avagyan, V., Ma, N., Papadopoulos, J., Bealer, K., and Madden, T. L. (2009). Blast+: architecture and applications. BMC bioinformatics, 10(1):421.

Castanheira, M., Deshpande, L. M., Mathai, D., Bell, J. M., Jones, R. N., and Mendes, R. E. (2011). Early dissemination of ndm-1-and oxa-181-producing enterobacteriaceae in indian hospitals: report from the sentry antimicrobial surveillance program, 2006-2007. Antimicrobial agents and chemotherapy, 55(3):1274-1278.

Chen, Y., Zhen, Q., Wang, Y., Xu, J., Sun, Y., Li, T., Gao, L., Guo, F., Wang, D., Yuan, X., et al. (2011). Development of an extended multilocus sequence typing for genotyping of brucella isolates. Journal of microbiological methods, 86(2):252-254.

Crisafulli, G., Guidotti, S., Muzzi, A., Torricelli, G., Moschioni, M., Masignani, V., Censini, S., and Donati, C. (2013). An extended multi-locus molecular typing schema for streptococcus pneumoniae demonstrates that a limited number of capsular switch events is responsible for serotype heterogeneity of closely related strains from different countries. Infection, Genetics and Evolution, 13:151-161.

Csardi, G. and Nepusz, T. (2006). The igraph software package for complex network research. InterJournal, Complex Systems, 1695(5):1-9.

Dingle, K. E., McCarthy, N. D., Cody, A. J., Peto, T. E., and Maiden, M. C. (2008). Extended sequence typing of campylobacter spp., united kingdom. Emerging infectious diseases, 14(10):1620.

Gupta, A., Jordan, I. K., and Rishishwar, L. (2016). stringmlst: a fast k-mer based tool for multilocus sequence typing. Bioinformatics, 33(1):119-121.

Iraola, G., Forster, S. C., Kumar, N., Lehours, P., Bekal, S., García-Peña, F. J., Paolicchi, F., Morsella, C., Hotzel, H., Hsueh, P.-R., et al. (2017). Distinct campylobacter fetus lineages adapted as livestock pathogens and human pathobionts in the intestinal microbiota. Nature Communications, 8(1):1367. 
Jolley, K. A., Bliss, C. M., Bennett, J. S., Bratcher, H. B., Brehony, C., Colles, F. M., Wimalarathna, H., Harrison, O. B., Sheppard, S. K., Cody, A. J., et al. (2012). Ribosomal multilocus sequence typing: universal characterization of bacteria from domain to strain. Microbiology, 158(4):1005-1015.

Jolley, K. A. and Maiden, M. C. (2010). Bigsdb: scalable analysis of bacterial genome variation at the population level. BMC bioinformatics, 11(1):595.

Larsen, M. V., Cosentino, S., Rasmussen, S., Friis, C., Hasman, H., Marvig, R. L., Jelsbak, L., SicheritzPontén, T., Ussery, D. W., Aarestrup, F. M., et al. (2012). Multilocus sequence typing of total-genomesequenced bacteria. Journal of clinical microbiology, 50(4):1355-1361.

Liu, L., Li, Y., Li, S., Hu, N., He, Y., Pong, R., Lin, D., Lu, L., and Law, M. (2012). Comparison of next-generation sequencing systems. BioMed Research International, 2012.

Maiden, M. C., Bygraves, J. A., Feil, E., Morelli, G., Russell, J. E., Urwin, R., Zhang, Q., Zhou, J., Zurth, K., Caugant, D. A., et al. (1998). Multilocus sequence typing: a portable approach to the identification of clones within populations of pathogenic microorganisms. Proceedings of the National Academy of Sciences, 95(6):3140-3145.

Maiden, M. C., Van Rensburg, M. J. J., Bray, J. E., Earle, S. G., Ford, S. A., Jolley, K. A., and McCarthy, N. D. (2013). Mlst revisited: the gene-by-gene approach to bacterial genomics. Nature Reviews Microbiology, 11(10):728.

Page, A. J., Alikhan, N.-F., Carleton, H. A., Seemann, T., Keane, J. A., and Katz, L. S. (2017). Comparison of classical multi-locus sequence typing software for next-generation sequencing data. Microbial genomics, 3(8).

Page, A. J., Taylor, B., and Keane, J. A. (2016). Multilocus sequence typing by blast from de novo assemblies against pubmlst. The Journal of Open Source Software, 1(8).

Paradis, E., Claude, J., and Strimmer, K. (2004). Ape: analyses of phylogenetics and evolution in $\mathrm{r}$ language. Bioinformatics, 20(2):289-290.

R Development Core Team (2008). R: A Language and Environment for Statistical Computing. R Foundation for Statistical Computing, Vienna, Austria. ISBN 3-900051-07-0.

Urwin, R. and Maiden, M. C. (2003). Multi-locus sequence typing: a tool for global epidemiology. Trends in microbiology, 11(10):479-487. 